\title{
Risk Factors for Drug-Resistant Tuberculosis
}

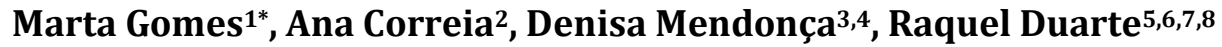 \\ ${ }^{1}$ Occupational Health, Centro Hospitalar Vila Nova de Gaia/Espinho EPE, Vila Nova de Gaia, Portugal \\ ${ }^{2}$ Public Health Department, Northern Regional Health Administration, Porto, Portugal \\ ${ }^{3}$ Institute of Public Health, University of Porto, Porto, Portugal \\ ${ }^{4}$ Institute of Biomedical Sciences Abel Salazar, Porto, Portugal \\ ${ }^{5}$ Chest Disease Centre Vila Nova de Gaia, Vila Nova de Gaia, Portugal \\ ${ }^{6}$ Pulmonology, Centro Hospitalar de Vila Nova de Gaia/Espinho EPE, Vila Nova de Gaia, Portugal \\ ${ }^{7}$ Reference Centre for Multidrug Resistant Tuberculosis, Northern Regional Health Administration, Porto, Portugal \\ ${ }^{8}$ Department of Clinical Epidemiology, Predictive Medicine and Public Health, University of Porto Medical \\ School, Porto, Portugal \\ Email: marta.gomes@chvng.min-saude.pt
}

Received 13 June 2014; revised 23 July 2014; accepted 5 August 2014

Copyright (C) 2014 by authors and Scientific Research Publishing Inc.

This work is licensed under the Creative Commons Attribution International License (CC BY).

http://creativecommons.org/licenses/by/4.0/

(c) (i) Open Access

\section{Abstract}

Objective: Drug resistance is considered one of the main threats for tuberculosis control. Our aim was to identify risk factors for drug resistance in tuberculosis patients in the Northern Portugal. Study Design and Methods: Retrospective case-control study. The medical records and drug susceptibility test data from TB patients diagnosed between 31 March 2009 and 1 April 2010 were examined. We enrolled 119 patients with any drug resistance to first line anti-TB drugs and 238 with drug-susceptible TB, matched by age group. Variables analyzed included: gender, country of origin, employment situation, site of disease, previous treatment, presence of diabetes mellitus, HIV infection, alcohol abuse, intravenous drug use, abuse of other drugs and smoking habits. Multivariate conditional logistic regression was used to identify independent predictors for drug-resistant TB. Results: Diabetes mellitus [adjusted odds ratio (OR): 3.54; 95\% CI: 1.45 - 8.66], intravenous drug use (OR: 4.77; 95\% CI: 1.24 - 18.32) and previous TB treatment (OR: 2.48; 95\% CI: 1.12 - 5.49) were found to be risk factors for drug-resistant disease development. Conclusions: Diabetes mellitus, prior tuberculosis treatment, and intravenous drug use were risk factors for drug-resistant disease. The association between diabetes and drug-resistant TB should be further explored. Identifying clinical predictors of drug resistance can allow prompt identification of patients at risk for drug-resistant TB.

\footnotetext{
*Corresponding author.
} 


\section{Keywords}

\section{Tuberculosis, Drug-Resistance, Risk, Diabetes, Portugal}

\section{Introduction}

Drug-resistant tuberculosis (TB) is a worldwide threat and constitutes an unparalleled challenge for disease control. Several studies have demonstrated worse clinical outcomes in patients diagnosed with drug-resistant TB [1]. Treatment of such patients is more complex, less effective, more toxic, and much more expensive than treatment of patients infected with drug-susceptible TB strains [1].

In 2008, the World Health Organization (WHO) estimated that more than 440,000 individuals had multidrugresistant tuberculosis (MDR-TB) worldwide. Such patients are resistant to at least isoniazid and rifampicin, the most effective anti-TB drugs. MDR-TB patients constituted 3.6\% of all TB patients registered globally [2].

Almost 50\% of MDR-TB cases are estimated to occur in China and India [1]. In Europe, in 2009, the European Centre for Disease Prevention and Control estimated a proportion of $14.6 \%$ cases resistant to one or more first line anti-TB drugs (rifampicin, isoniazid, ethambutol, and streptomycin) and MDR-TB patients constituted $5.3 \%$ of all registered cases of TB [3].

Portugal is considered as having an intermediate incidence of TB (22 cases/100,000 in 2010) and the risk of disease has been consistently decreasing over the last 10 years [4]. In 2009, the proportion of cases resistant to one or more first line anti-TB drugs was $13.8 \%$ of all TB cases registered in Portugal. Resistance to isoniazid (predictor for the development of MDR-TB) was low (6.8\% of all new cases). The overall proportion of MDRTB (1.5\%) is comparable with the median incidence in Europe. However, in some areas (Lisbon and Porto) MDR-TB is endemic. Another matter of concern is that the proportion of MDR-TB patients that are extensively drug-resistant (XDR-TB) (thus having the poorest outcomes because virtually untreatable) is higher in Portugal (32\%) than in Europe (7\%) [4]-[6].

International studies have identified various risk factors for drug-resistant TB; these include previous TB treatment, poor adherence to treatment regimens, inadequate regimens and positive smear result at the end of the second and third month of treatment [7]-[17]. In addition of these programmatic factors, patients characteristics such as HIV co-infection, alcohol abuse and younger age [7]-[14] are also believe to influence the drug resistance. However, information available on some of these issues is limited and controversial. For instance, high prevalence levels of drug resistance have been found among HIV patients [11]-[13]. However, other studies have found no association between HIV and drug resistance [9].

The age is accepted by WHO as a risk factor for drug resistance [18]. Guidelines for surveillance of drug resistance in tuberculosis suggest data on drug resistance stratified by age groups. This fact can provide insight into risk groups and effectiveness of specifics TB control activities [18]. Furthermore, the magnitude of drug resistance among younger age is more likely to be indicative of recent transmission than among older age groups, which are more likely to be harbouring older infections [18].

Although in Portugal resistance to anti-TB drugs is considered a priority, only few studies have focused on the relevant risk factors.

The aim of the present study was to identify risk factors for drug resistance in TB patients in northern Portugal.

\section{Methods}

\subsection{Setting}

In Portugal, all patients with tuberculosis are treated under a National TB Control Program (NTP); patients are treated in specialized centers (Tuberculosis Units), medication is available through the NTP, is free of charge and is directly observed. Drug susceptibility testing (proportion method) is performed on every TB patient with positive Mycobacterium tuberculosis (Mt) culture before treatment [6].

\subsection{Design and Patients}

We performed a retrospective case-control study. The medical records and the DST results for all patients diag- 
nosed with and registered as having TB, from 31 March 2009 until 1 April 2010 in the north of Portugal, were reviewed.

The clinician notifies all tuberculosis cases (national level) and the laboratory mandatorily notifies all DST at the moment of diagnosis (a pilot project in the northern region since 2009). This data is crosschecked and quality of data is evaluated at regional level and later at national level.

Cases and suitable controls were identified after the crosscheck of both databases. All patients resistant to one or more first-line anti-TB drugs (isoniazid, rifampicin, ethambutol and streptomycin) were classified as cases. For each case we randomly identified two age group-matched patients (controls) showing susceptibility to all first-line drugs. The ratio cases-controls possible to include all the cases was 1/2. All selected controls had already concluded the treatment. Age was categorized into five groups: 0 - 14 years, 15 - 24 years, 25 - 44 years, 45 - 64 years, and 65 years or older.

We reviewed medical records of all patients selected as cases and controls. Demographic characteristics and risk factor information were routinely and mandatory obtained for each patient on admission to a Tuberculosis Unit. This information was collected using a structured questionnaire and stored in the National Tuberculosis Surveillance System (SVIG-TB). National guidelines determine that HIV testing should be offered to all patients unless a previous positive HIV test result has been recorded.

The variables under analysis included gender, place of birth (Portugal-born or foreign-born), employment situation (employed or unemployed), site of disease (pulmonary or extra-pulmonary), previous treatment (defined as treatment with anti-TB drugs for more than 1 month), diabetes mellitus, HIV infection, alcohol abuse, intravenous drug use, abuse of other drugs and smoking habits (current smoker). The comorbidities included in the analysis preceded diagnosis of DRTB.

\subsection{Statistical Analysis}

The measure of association between drug resistance and each potential risk factor was reported by odds ratio (OR) and the 95\% confidence intervals (95\% CI). Initially, each potential risk factor was evaluated using univariate conditional logistic regression models. Then, based on these results, a multivariate conditional logistic regression model was constructed including all factors with a $\mathrm{p}<0.07$ in the univariate analysis.

Statistical analysis was performed using STATA (Statistics Data Analysis), Version 9.

\section{Results}

During the studied period, 886 patients were diagnosed with TB. Drug-resistant TB bacilli were found in 129 patients (14.6\%). Ten of these patients were excluded because there was no clinical information available. Table 1 shows the resistance patterns among all 876 patients diagnosed with TB. The results for pirazinamide susceptibility were not available.

\begin{tabular}{cc} 
Table 1. Resistance pattern among 876 patients diagnosed with TB. \\
\hline Resistance pattern & $\mathrm{n}(\%)$ \\
\hline Fully sensitive & $757(86.4)$ \\
Any resistance & $119(13.6)$ \\
Resistance to & \\
Only isoniazid (H) & $12(1.4)$ \\
Only rifampicin (R) & $2(0.2)$ \\
Only ethambutol (E) & 0 \\
Only streptomycin (S) & $64(7.3)$ \\
ES & $1(0.1)$ \\
HR & $10(1.1)$ \\
HS & $29(3.3)$ \\
HSE & $1(0.1)$ \\
Any H resistance & $52(5.9)$ \\
Any R resistance & $12(1.4)$ \\
Any E resistance & $2(0.2)$ \\
Any S resistance & $95(10.8)$ \\
\hline
\end{tabular}


We enrolled 119 patients with drug-resistant TB and 238 controls with drug-susceptible disease. In the case group, the mean age was 48.5 years (SD 17.3 years) and $65.5 \%(n=78)$ were male. One hundred and one patients $(84.9 \%)$ had symptoms at the time of diagnosis. Sixteen patients $(13.4 \%)$ were detected by risk groups screening activities and 2 patients (1.7\%) by contact investigation.

In the control group, mean patient age was 48.2 years (SD 18.2 years) and $64.3 \%(n=153)$ were male.

Among all patients with previous treatment, only one has been considered as a treatment default. All the others had treatment complete.

Overall, three patients were homeless, two had history of imprisonment and three lived in community housing. These variables were not included in the analysis due to its low frequency. Data on time for sputum smear conversion were also not included due to missing information.

Table 2 shows the results of univariate analysis of potential risk factors. The variables associated with drug-

Table 2. Predictors for drug resistant TB among 357 tuberculosis patients.

\begin{tabular}{|c|c|c|c|c|c|}
\hline Variables & $\begin{array}{c}\text { Cases } \\
\mathrm{n}(\%)(\mathrm{N}=119)\end{array}$ & $\begin{array}{c}\text { Controls } \\
\text { n (\%) }(\mathrm{N}=238)\end{array}$ & $\mathrm{P}$ value & $\begin{array}{l}\text { Unadjusted OR } \\
\text { (95\% CI) }\end{array}$ & $\begin{array}{l}\text { Adjusted OR } \\
(95 \% \mathrm{CI})\end{array}$ \\
\hline \multicolumn{6}{|l|}{ Gender } \\
\hline Male & 78 (65.5) & 153 (64.3) & & Reference & \\
\hline Female & $41(34.5)$ & $85(35.7)$ & 0.80 & $0.94(0.58-1.53)$ & \\
\hline \multicolumn{6}{|l|}{ Country of origin } \\
\hline Portugal & $114(95.8)$ & $232(97.5)$ & & Reference & \\
\hline Foreign-born & $5(4.2)$ & $6(2.5)$ & 0.40 & $1.67(0.51-5.46)$ & \\
\hline \multicolumn{6}{|c|}{ Employment situation } \\
\hline Employed & $53(48.2)$ & $103(45.0)$ & & Reference & \\
\hline Unemployed & $57(51.8)$ & $126(55.0)$ & 0.75 & $0.92(0.54-1.56)$ & \\
\hline \multicolumn{6}{|l|}{ Site of disease } \\
\hline Pulmonary & $104(87.4)$ & $207(87.0)$ & & Reference & \\
\hline Extra-pulmonary & $15(12.6)$ & $31(13.0)$ & 0.91 & $0.96(0.51-1.84)$ & \\
\hline \multicolumn{6}{|l|}{ Previous treatment } \\
\hline No & $98(85.2)$ & 222 (93.3) & & Reference & \\
\hline Yes & $17(14.8)$ & $16(6.7)$ & 0.02 & $2.52(1.19-5.34)$ & $2.48(1.12-5.49)$ \\
\hline \multicolumn{6}{|l|}{ Co morbidities } \\
\hline \multicolumn{6}{|l|}{ Diabetes } \\
\hline No & 105 (88.2) & 228 (95.8) & & Reference & \\
\hline Yes & $14(11.8)$ & $10(4.2)$ & 0.01 & $2.97(1.28-6.89)$ & $3.54(1.45-8.66)$ \\
\hline \multicolumn{6}{|l|}{ HIV infection } \\
\hline No & 88 (86.3) & 183 (89.3) & & Reference & \\
\hline Yes & $14(13.7)$ & $22(10.7)$ & 0.36 & $1.48(0.64$ - 3.39) & \\
\hline \multicolumn{6}{|l|}{ Behaviour factors } \\
\hline \multicolumn{6}{|l|}{ Alcohol abuse } \\
\hline No & $100(84.0)$ & $200(84.7)$ & & Reference & \\
\hline Yes & $19(16.0)$ & $36(15.3)$ & 0.88 & $1.05(0.57-1.95)$ & \\
\hline \multicolumn{6}{|l|}{ Intravenous drugs } \\
\hline No & $106(89.8)$ & 227 (95.8) & & Reference & \\
\hline Yes & $12(10.2)$ & $10(4.2)$ & 0.01 & $5.30(1.42-19.70)$ & $4.77(1.24-18.32)$ \\
\hline \multicolumn{6}{|l|}{ Other drugs abuse } \\
\hline No & $111(93.3)$ & $223(94.1)$ & & Reference & \\
\hline yes & $8(6,7)$ & $14(5.9)$ & 0.72 & $1.22(0.42-3.52)$ & \\
\hline \multicolumn{6}{|l|}{ Current smoker } \\
\hline No & $118(99.2)$ & 232 (97.5) & & Reference & \\
\hline Yes & $1(0.8)$ & $6(2.5)$ & 0.27 & $0.29(0.03-2.65)$ & \\
\hline
\end{tabular}


resistant TB were previous TB treatment, presence of diabetes mellitus, and intravenous drug use. Patients previously treated for TB now have significantly increased odds of drug-resistant TB compared to newly diagnosed patients (OR = 2.52; 95\% CI: $1.19-5.34 ; \mathrm{p}=0.02)$. Diabetic patients were more likely to have drug-resistant TB than non-diabetic patients ( $\mathrm{OR}=2.97 ; 95 \% \mathrm{CI}: 1.28-6.89 ; \mathrm{p}=0.01)$. Patients who used intravenous drugs have significantly higher odds of drug-resistant $\mathrm{TB}$ than those who did not use such drugs (OR $=5.30$; $95 \% \mathrm{CI}$ : 1.42 - 19.70; $\mathrm{p}=0.01)$. A higher proportion of HIV-positive patients was evident among cases $(13.7 \%)$ compared to the control group (10.7\%). However, these values were not significantly different and HIV co-infection was not a significant risk factor for drug-resistant TB.

Multivariate analysis showed that the potential risk factors showing significant associations with drug-resistant TB in univariate analysis remained independently associated with drug-resistant TB. In the final multivariable model, previous TB treatment (adjusted OR: 2.48; 95\% CI: 1.12 - 5.49), presence of diabetes mellitus (adjusted OR: 3.54; 95\% CI: 1.45 - 8.66), and use of intravenous drugs (adjusted OR: 4.77; 95\% CI: 1.24 - 18.32) were all significant risk factors for drug-resistant TB.

\section{Discussion}

The factors independently associated with drug-resistant TB were diabetes mellitus, intravenous drug use and previous TB treatment.

A link between diabetes mellitus and TB has been recognized for centuries. There is growing evidence that diabetes mellitus is an important risk factor for TB [19]-[23] and might affect disease presentation and treatment response [20] [24]. WHO estimates that diabetes contribute to about $8 \%$ of new cases annually [22]. In a setting of increasing overlap of populations at risk for either disease, the combination of TB and diabetes mellitus represents a worldwide health threat [20]. The number of patients with diabetes, 171 million in 2000, is expected to grow to 366 - 440 million by 2030 [20] [21], with almost $80 \%$ of diabetic patients living in low-and-middleincome countries where most TB is found [21].

The mechanism by which diabetes can cause development of drug-resistant TB is unclear. Possible malabsorption of anti-TB drugs among diabetic patients, thus reducing the treatment effect, has been suggested [20] [24]. Just as tuberculosis drug treatment affects diabetes treatment, diabetes might alter the pharmacokinetics of antituberculosis drugs. Diabetes can cause changes in oral absorption, decreased protein binding of drugs and renal insufficiency or fatty liver with impaired drug clearance [20]. Fatty liver may be associated with hepatic toxicity that can potentially lead to sub-therapeutic drug levels and acquired DR [21]. Diabetes may affect TB treatment outcome by delaying time to microbiological response [20] [24]. Thus, it is possible that resistance develops because diabetic patients do not get treatment for long enough.

As we do not have genotyping information, we cannot exclude that diabetic patients may be more susceptible to disease following infection with resistant strains.

Given the rising incidence of diabetes in patients living in low-and-middle income countries, the management of diabetes in TB patients would improve TB treatment outcome and reduce the risk of recurrent TB.

Our results suggest that intravenous drug users are more likely to have drug-resistant TB than individuals who do not inject drugs. Intravenous drug use was also identified as a significant risk factor by other authors [12] [25]. Possible explanations include late diagnosis, poor compliance with treatment, and treatment default [26] [27]. Compliance is known to be problematic in such groups [26] [27]. Studies have demonstrated the deleterious effects of drug use on the immune system [27]. This fact, associated with the physiological effects of drug use and the risk behaviors of drug users may all contribute to development of poorer outcomes. Together, these physiological and epidemiologic factors may each contribute to observe that drug users are more likely to take longer to achieve negative culture [27], drug resistance rises over time and the mortality risk is increased [27].

Another possible explanation for the association between intravenous drug use and drug resistant TB could be related to close contacts of drug users and transmission of resistant strains within this group [25] [27]. As we do not have genotyping data available, we cannot exclude the existence of possible clusters. However, in our study only one patient was identified due to contact screening among intravenous drug users.

Previous TB treatment, a well-known risk factor for drug-resistant TB [7] [9]-[11] [14] [15] was also found to be significantly associated with drug resistance in the present study. Although drug-resistant TB can be transmitted between individuals (primary resistance), most cases arise after inadequate treatment allowing a drug-resistant strain to become dominant [10]. The use of directly observed therapy (DOT) is highly recommended to 
promote treatment adherence [16] [26]. Recent studies suggest that resistance is less likely to develop or to be transmitted when TB patients are under DOT [16] [28].

We did not find a significant association between HIV co-infection and drug-resistance, although this association has been described [11]-[13]. Lack of such an association could be caused by inadequate testing [2], but in Portugal, in 2009, HIV testing was performed on $87 \%$ of all TB patients and HIV co-infection prevalence was 15\% [2]. More information about characteristics of HIV-infected patients, namely mean of CD4 count, anti-retroviral therapy is needed to understand this result.

In our study, there was a low frequency of immigration. This information was verified with patient's identification documents. This could be explained by the economic and social conditions in the country with high rates of unemployment.

This investigation had some limitations. Because of our retrospective design, we could analyze only the risk factor data that were routinely recorded in Tuberculosis Units.

However, considering the similar age distribution of cases and controls, we can assume that no relevant selection bias was likely to occur. We cannot exclude the occurrence of information bias and misclassification because information about co-morbidities, namely about smoking habits, alcohol abuse or diabetes, was based on patient information and was not laboratory confirmed. We must consider that TB patients could under report co-morbidities, but we have no reasons to believe that under reporting would be different among drug resistant and drug susceptible cases. Information about behavior factors was based on clinical judgment, which could originate both under and over reporting.

The association between diabetes and drug resistance could be explained by confounding factors, namely by obesity or nephropathy. We were not able to test the effect of obesity or nephropathy as confounders because this information is not collected.

In spite of limitations, our study provides relevant initial data about drug resistance in Portugal. To the best of our knowledge, the present case-control study is the first investigation of risk factors for drug-resistant TB in the Northern Region of Portugal.

\section{Conclusion}

The present findings associated drug-resistant TB with diabetes mellitus, previous TB treatment and intravenous drug use. Knowledge of these factors conferring risk permits identification of patients with a predisposition to development of drug resistance, thus allowing efficacious treatment regimens to be implemented. The fact that diabetes mellitus is a risk factor for drug-resistant TB development represents an enormous threat to TB control. This association should be further explored to effectively co-manage the two diseases.

\section{Acknowledgements}

The authors have no funding or conflict of interest.

This investigation was approved by the Regional Ethical Committee and the Northern Regional Coordination for NPT.

\section{Author's Contribution}

MG, AC, DM and RD had substantial contribution to conception, design of the study, analysis and interpretation of data. MG did the acquisition of data, and drafting the article. RD and AC revised the article critically for intellectual content. All the authors gave final approval of the version to be published.

\section{References}

[1] World Health Organization (WHO) (2010) The Global Plan to Stop TB 2011-2015. World Health Organization, Geneva.

[2] World Health Organization (WHO) (2010) Multidrug and Extensively Drug-Resistant TB (M/XDR-TB)-2010. Global Report on Surveillance and Response. World Health Organization, Geneva.

[3] European Centre for Disease Prevention and Control/World Health Organization Regional Office for Europe (2014) Tuberculosis Surveillance in Europe 2009. Stockholm, European Centre for Disease Prevention and Control, 2011. http://www.ecdc.europa.eu 
[4] Direcção Geral de Saúde (2011) Programa Nacional de Luta contra a Tuberculose. Ponto de situação epidemiológica e de desempenho. Divisão de Doenças Transmissíveis.

[5] Administração Regional da Saúde no Norte, I.P. A Tuberculose na Região de Saúde do Norte 2000-2010. Ano 2011. Unidade de Vigilância Epidemiológica.

[6] Programa Nacional de Luta contra a Tuberculose. Perfil de susceptibilidade aos antibióticos de estirpe Mycobacterium Tuberculosis—Sistema de notificação laboratorial 2009. Ano 2010. Administração Regional de Saúde do Norte.

[7] Yang, X., Li, Y., Wen, X., Wu, X. and Li, X. (2010) Risk Factors for Drug Resistance in Pulmonary Tuberculosis in Patients. JEBM, 3, 162-167.

[8] Caminero, J.A. (2010) Multidrug-Resistant Tuberculosis: Epidemiology, Risk Factors and Case Finding. International Journal of Tuberculosis and Lung Disease, 14, 382-390.

[9] Farazi, A., Sofian, M., Zarrinfan, N., Katebi, F., Hoseini, S. and Keshavarz, R. (2013) Drug Resistance Patterns and Associated Risk Factors of Tuberculosis in the Central Province of Iran. Caspian Journal of Internal Medicine, 4, 785-789.

[10] Suárez-Garcia, I., Rodrígues-Blanco, A., Vidal-Pérez, J.L., García-Viejo, M.A., Jaras-Hernandez, M.J., López, O., et al. (2009) Risk Factors for Multidrug-Resistant Tuberculosis in a Tuberculosis Unit in Madrid, Spain. European Journal of Clinical Microbiology Infectious Diseases, 28, 325-330. http://dx.doi.org/10.1007/s10096-008-0627-y

[11] Faustini, A., Hall, A.J. and Perucci, C.A. (2006) Risk Factors for Multidrug Resistant Tuberculosis in Europe: A Systematic Review. Thorax, 61, 158-163. http://dx.doi.org/10.1136/thx.2005.045963

[12] Casal, M., Vaquero, M., Rinder, H., Tortoli, E., Grosset, J., Rusch-Gerdes, S., et al. (2005) A Case-Control Study for Multidrug-Resistant Tuberculosis: Risk Factors in Four European Countries. Microbial Drug Resistance, 11, 62-67. http://dx.doi.org/10.1089/mdr.2005.11.62

[13] Kliiman, M.D. and Altraja, A. (2009) Predictors of Extensively Drug-Resistant Pulmonary Tuberculosis. Annals of Internal Medicine, 150, 766-775. http://dx.doi.org/10.7326/0003-4819-150-11-200906020-00004

[14] Lomtadze, N., Aspindzelasvili, R., Janjgava, M., Mirtskhulava, V., Wright, A., Blumberg, H.M. and Salakaia, A. (2009) Prevalence and Risk Factors for Multidrug-Resistant Tuberculosis in Republic of Georgia: A Population Based Study. The International Journal of Tuberculosis and Lung Disease, 13, 68-73.

[15] Liu, Q., Zhu, L., Shao, Y., Song, H., Li, G., Zhou, Y., et al. (2013) Rates and Risk Factors for Drug Resistance Tuberculosis in Northeastern China. BMC Public Health, 13, 1171. http://dx.doi.org/10.1186/1471-2458-13-1171

[16] Flora, M.S., Amin, M.N., Karim, M.R., Afros, S., Islam, S., Alam, A., et al. (2013) Risk Factors of Multi-Drug-Resistant Tuberculosis in Bangladeshi Population: A Case Control Study. Bangladesh Medical Research Council Bulletin, 39, 34-41. http://dx.doi.org/10.3329/bmrcb.v39i1.15808

[17] Wang, K., Chen, S., Wang, X., Zhong, J., Wang, X., Huai, P., et al. (2014) Factors Contributing to the High Prevalence of Multidrug-Resistant Tuberculosis among Previously Treated Patients: A Case-Control Study from China. Microbial Drug Resistance, 20, 294-300.

[18] World Health Organization. (2009) Guidelines for Surveillance of Drug Resistance in Tuberculosis. 4th Edition, WHO, Geneva.

[19] Alisjahbana, B., Sahiratmadja, E., Nelwan, E.J., Purwa, A.M., Ahmad, Y., Ottenhoff, T.H., et al. (2007) The Effect of Type 2 Diabetes Mellitus on the Presentation and Treatment Response of Pulmonary Tuberculosis. Clinical Infectious Diseases, 45, 428-435. http://dx.doi.org/10.1086/519841

[20] Dooley, K. and Chaisson, R. (2009) Tuberculosis and Diabetes Mellitus: Convergence of Two Epidemics. The Lancet Infectious Diseases, 9, 737-746. http://dx.doi.org/10.1016/S1473-3099(09)70282-8

[21] Harries, A., Lin, Y., Satyanarayana, S., Lonnroth, K., Li, L., Wilson, N., et al. (2011) The Looming Epidemic of Diabetes-Associated Tuberculosis: Learning Lessons from HIV-Associated Tuberculosis. The International Journal of Tuberculosis and Lung Disease, 15, 1436-1444. http://dx.doi.org/10.5588/ijtld.11.0503

[22] Maurice, J. (2011) WHO Framework Target Tuberculosis-Diabetes Link. The Lancet, 378, 1209-1210. http://dx.doi.org/10.1016/S0140-6736(11)61527-4

[23] Baker, M., Harries, A., Jeon, C., Hart, J., Kapur, A., Lonnroth, K., et al. (2011) The Impact of Diabetes on Tuberculosis Treatment Outcomes: A Systematic Review. BMC Medicine, 9, 81. http://dx.doi.org/10.1186/1741-7015-9-81

[24] Mi, F., Tan, S., Liang, L., Harries, A., Hinderaker, S., Lin, Y., et al. (2013) Diabetes Mellitus and Tuberculosis: Patterns of Tuberculosis, Two-Month Smear Conversion and Treatment Outcomes in Guangzhou, China. Tropical Medicine and International Health, 18, 1379-1385. http://dx.doi.org/10.1111/tmi.12198

[25] Mdivani, N., Zangaladze, E., Volkova, N., Kourbatova, E., Jibuti, T., Shubladze, N., et al. (2008) High Prevalence of Multidrug-Resistant Tuberculosis in Georgia. International Journal of Infectious Diseases, 12, 635-644. http://dx.doi.org/10.1016/j.ijid.2008.03.012 
[26] Duarte, R., Santos, A., Mota, M., Carvalho, A., Marques, A. and Barros, H. (2011) Involving Community Partners in the Management of Tuberculosis among Drug Users. Public Health, 125, 60-62. http://dx.doi.org/10.1016/j.puhe.2010.09.002

[27] Deiss, R., Rodwell, T. and Garfein, R. (2009) Tuberculosis and Drug Use: Review and Update. Clinical Infectious Diseases, 48, 72-82. http://dx.doi.org/10.1086/594126

[28] Moonan, P., Quituga, T., Pogoda, J., Woo, G., Drewyer, G., Sahbazian, B., et al. (2011) Does Directly Observed Therapy (DOT) Reduce Drug Resistant Tuberculosis? BMC Public Health, 11, 19. http://dx.doi.org/10.1186/1471-2458-11-19 
Scientific Research Publishing (SCIRP) is one of the largest Open Access journal publishers. It is currently publishing more than 200 open access, online, peer-reviewed journals covering a wide range of academic disciplines. SCIRP serves the worldwide academic communities and contributes to the progress and application of science with its publication.

Other selected journals from SCIRP are listed as below. Submit your manuscript to us via either submit@scirp.org or Online Submission Portal.
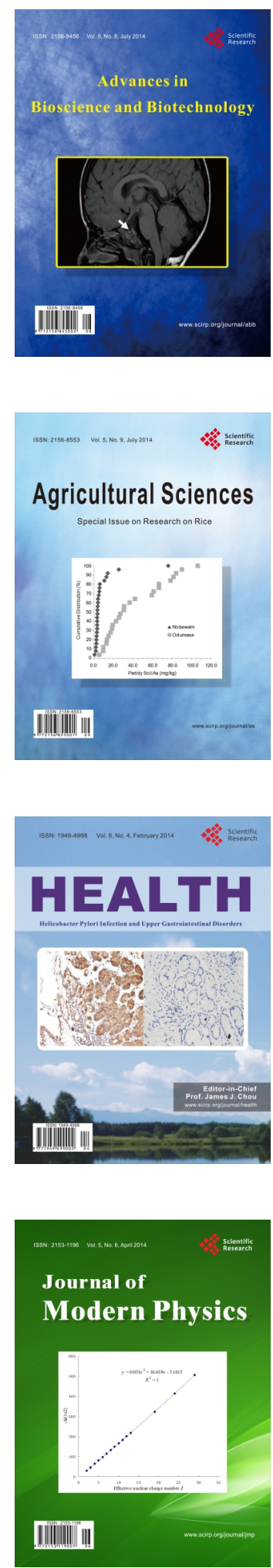
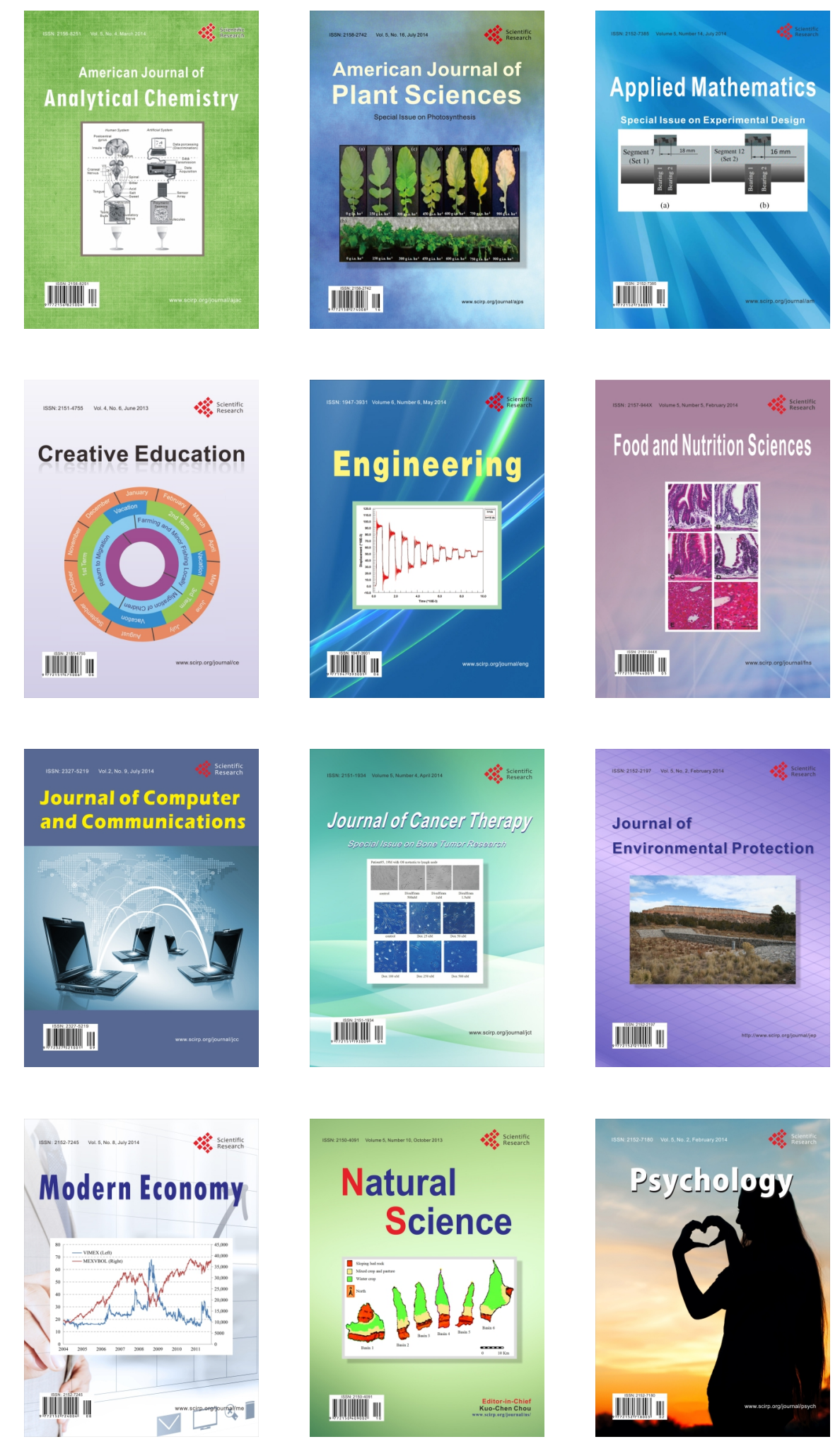